\title{
Scalable Generation of Graph-State Entanglement through Realistic Linear Optics
}

\author{
T. P. Bodiya, L.-M. Duan \\ FOCUS Center and MCTP, Department of Physics, University of Michigan, Ann Arbor, MI 48109
}

\begin{abstract}
We propose a scheme for efficient construction of graph states using realistic linear optics, imperfect photon source and single-photon detectors. For any many-body entanglement represented by tree graph states, we prove that the overall preparation and detection efficiency scales only polynomially with the size of the graph, no matter how small the efficiencies for the photon source and the detectors.
\end{abstract}

Linear optics, combined with practical photon source and single-photon detectors, has provided a powerful tool to test a number of quantum information protocols [1, 2, 3, 4, 5]. In the linear optics implementations, the post-selection technique based on photon detections typically plays a critical role. As one scales up the system, the post-selection technique generally leads to very inefficient (exponential) scaling of the overall efficiency, which limits the implementation to small systems. In the past few years, two approaches have been proposed to circumvent this obstacle for different kinds of quantum information processing. In the first approach, a remarkable proposal, generally referred to as linear optics quantum computation, was first put forward by Knill, Laflamme, and Milburn (KLM) 6] and then improved by a number of others $\mathbf{7}, 8,9,10,11$. In this approach, one overcomes the scaling problem for linear optics computation through quantum error correction by requiring the efficiencies for the photon source and the detectors to attain a high threshold value [6]. The threshold efficiency has been improved considerably in the past few years, with the most recent estimate about $99.4 \%$ - $99.7 \%$ 11]. In the second approach proposed in Ref. 12], one uses linear optics for implementation of scalable quantum communication through the quantum repeater protocol 13. An extension of that protocol can also efficiently prepare GHZ type of entanglement 14]. This approach overcomes the inefficient scaling through the divide-andconquer method. In this protocol, one does not have a threshold requirement on the source and the detector efficiencies [15], which allows its implementation with the state-of-the-art photon detectors [16].

In this paper, we propose an efficient scheme for quantum state engineering with linear optics. We show how to generate many-qubit entanglement represented by graph states. Graph states have been generally identified as a useful resource for many quantum information protocols, including quantum computation, communication, and fundamental test of quantum mechanics [17, 18]. The main result from this work is twofold: first, we analyze the effect of a polarization beam splitter (PBS) in the Hilbert subspace postselected by the photon detections, and show that a single PBS actually represents a powerful gate for generating graph states of arbitrary shapes. Other types of gates have been proposed before for preparation of graph states $[7,8,[9,10,11$, among them the most efficient one up to now seems to be the fusion gate
9]. Compared with that one, we show that a PBS is a more efficient gate in the sense that it does not waste any photons for each gate operation 19. This improvement is desirable as in current experiments the number of photons is a precious resource. Second, a more important result here is a proposed method for scalable generation and detection of many-qubit entanglement represented by tree graph states, with the latter having a number of applications in recent quantum information protocols [10, 18, 20]. Here, by "scalable", we mean the overall efficiency for preparation of a large-scale entanglement with a tree-graph structure scales nearly polynomially with the number of qubits. We have this efficient scaling no matter how small the efficiencies for the photon source and the detectors. The scheme here thus well fits the status of the current experimental technology.

We assume to have an imperfect source of entangled photon pairs, which generates states of the following general form

$$
\rho_{s}=\left(1-\eta_{s}\right) \rho_{\mathrm{vac}}+\eta_{s}|\Psi\rangle_{12}\langle\Psi|,
$$

where $|\Psi\rangle_{12}=\left(|H H\rangle_{12}+|V V\rangle_{12}\right) / \sqrt{2}$ represents a photon pair entangled in the polarization states $|H\rangle,|V\rangle$; $\rho_{\mathrm{vac}}$ stands for the vacuum component with no photon for the modes 1 and 2 ; and $\eta_{s}$ is the source efficiency for producing the entangled photon pair. In experiments, the entangled photon source is typically provided through the process of spontaneous parametric down conversion (SPDC), where the source efficiency $\eta_{s}$ is a small number 1, 2, 3, 4]. The pair state (1) can also be generated from other experimental setups, such as from decay of a single dipole (which could be a single atom, ion, or a quantum dot) in free space or in a cavity [21, 22], or from decay of an collective excitation in an atomic ensemble [16]. In these cases, one mode of the entangled pair is typically represented by a matter qubit, which can be transferred later to a photon qubit after a controllable delay.

Now we show any graph state in the Hilbert subspace postselected by the photon detection can be generated from the pair states (1) through a series of PBS gates. An n-qubit graph state is defined as the co-eigenstate of $n$ independent stabilizer operators $S_{i}=X_{i} \prod_{j} Z_{j}$, where $i$ denotes qubit $i$ (each qubit is associated with a vertex of the graph), $j$ runs over all the neighbors of the qubit $i$, and $X_{i}, Z_{i}$ are simply the Pauli operators $\sigma_{x}$ and $\sigma_{z}$ for qubit $i$ [17, 18]. In a graph, the qubits $i$ and $j$ are called neighbors if they are connected with an edge. The 
graph state reduces to a cluster state if the corresponding graph is a periodic lattice 17].

To show construction of the graph states, first we need to analyze the effect of a PBS in the subspace postselected by the photon detection. For linear optics quantum information, all the photon modes will be eventually measured in an appropriate polarization basis by a single-photon detector. We are interested only in the measurement outcomes with one photon registered from each mode (its polarization can be arbitrary). So, by this final measurement, one postselects a Hilbert subspace, which we denote as $S$. We only need to find out the state evolution in this "physical" subspace $S$, as the state component outside $S$ has no influence on the final measurement of the polarization qubits.

For a $\mathrm{PBS}$, it transfers the photon if it in $H$ polarization and reflects it if it is in $V$ polarization. So, after the $\mathrm{PBS}$, the photons from the two incoming modes go to different sides (modes) if and only if both photons have the same polarization, either $H H$ or $V V$. Otherwise, they will go to the same sides with the other mode in the vacuum state, which is outside of the "physical" subspace $S$. So, within the subspace $S$, the effect of a PBS is to perform a projection on the input state, described by the projector

$$
P=|H H\rangle_{12}\langle H H|+| V V\rangle_{12}\langle V V| .
$$

This projection is equivalent to a measurement of the operator $Z_{1} Z_{2}$ on the two input qubits 1 and 2 , with the final state kept only under the measurement outcome "+1" $\left(|H H\rangle_{12}\right.$ and $|V V\rangle_{12}$ are eigenstates of $Z_{1} Z_{2}$ with an eigenvalue "+1"). So in the physical subspace $S$, a single PBS performs an effective $Z_{1} Z_{2}$ measurement gate with a success probability of $1 / 2$ (the probability to stay in the "physical" space $S$ after the PBS).

We start with two entangled pairs 1,2 and 3,4, each pair described by the state (1). In the subspace $S$, the effective state is then given by $|\Psi\rangle_{12}$, which can be transferred to a two-bit graph state with a straightforward Hadamard gate on one of the qubits. So, for the pairs 1,2 and 3,4 , we can assume them to have the stabilizer operators $X_{1} Z_{2}, X_{2} Z_{1}$ and $X_{3} Z_{4}, X_{4} Z_{3}$, respectively. If the qubits 2 and 3 pass through a PBS, the effective output state in the subspace $S$ is then stabilized by the operators $Z_{2} Z_{3}, X_{2} X_{3} Z_{1} Z_{4}, X_{2} Z_{1}, X_{4} Z_{3}\left(X_{2} X_{3} Z_{1} Z_{4}\right.$ is a multiplication of the previous stabilizers $X_{2} Z_{1}$ and $X_{3} Z_{4}$, and it remains unchanged after the PBS as it commutes with the effective measurement gate $Z_{2} Z_{3}$ ). With a straightforward Hadamard gate $X_{3} \leftrightarrow Z_{3}$ (implemented with a half-wave plate), the above four stabilizers transform to the standard stabilizers for the 4-bit star-shape graph state as shown in Fig. 1.

For convenience, we call a combination of a PBS and a single-bit Hadamard operation as the PBS gate (see Fig. 1). An extension of the above construction yields the following important result: the PBS gate always joins two pieces of graphs, independent of the shapes of the initial pieces. This result can be generally proven as

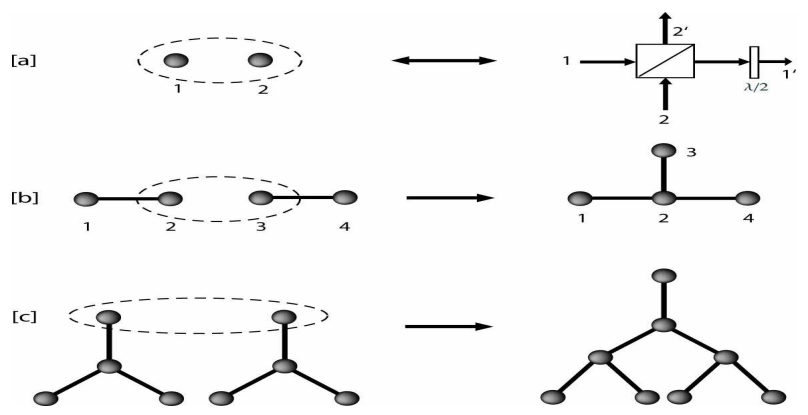

FIG. 1: [a]: The representation of the PBS gate, which consists of a polarization beam splitter and a half-wave plate (for a Hardamard operation on one mode). [b] and [c]: Illustration of using the PBS gates to generater tree graph states. It is obvious that tree graphs of any shapes can be generated with this method.
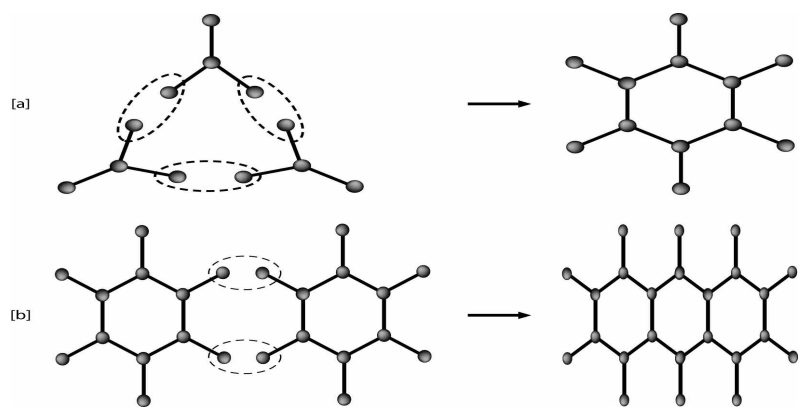

FIG. 2: [a] and [b]: Illustration of using the PBS gates to generate 2-dimensional graphs states.

follows: if we start with two pieces of graph states $G_{1}$ and $G_{2}$, with $n$ and $m$ qubits, respectively. The stabilizers associated with the qubits $i_{1}$ and $i_{2}$ are given by $S_{i_{l}}=X_{i_{l}} \prod_{j_{l} \in N\left(i_{l}\right)} Z_{j_{l}},(l=1,2)$, where $i_{l}$ is an arbitrary vertex of the graph $G_{l}$ and $N\left(i_{l}\right)$ denotes all the neighbors of the qubit $i_{l}$ in the graph $G_{l}$. After a PBS gate on the qubits $i_{1}$ and $i_{2}$, the stabilizers $S_{i_{2}}$ and $S_{i_{1}}$ are replaced by $S_{i_{2}}^{\prime}=X_{i_{2}} Z_{i_{1}}$ and $S_{i_{1}}^{\prime}=X_{i_{1}} Z_{i_{2}} \prod_{j_{1} \in N\left(i_{1}\right)} Z_{j_{1}} \prod_{j_{2} \in N\left(i_{2}\right)} Z_{j_{2}}$. All the other stabilizers of the initial graphs $G_{1}$ and $G_{2}$ remain unchanged after the gate. One can immediately see that the effective output state of the PBS gate is still a graph state which combines the two initial graphs $G_{1}$ and $G_{2}$, with $i_{2}$ attached to $i_{1}$, and $i_{1}$ attached to $i_{2}$ and all their initial neighbors in the graphs $G_{1}$ and $G_{2}$ (see Fig. $1[\mathrm{c}]$ ).

With the above result, it becomes possible to construct any shapes of the graph states with a series of PBS gates. In Figures 1 and 2, we illustrate the method by constructing the states represented respectively by a tree graph and by a two-dimensional graph with many loops. This construction method is efficient in the sense that no photon is wasted during the state preparation. Starting with $n$ entangled pairs, we get graph states of $2 n$ qubits with various shapes.

If we assume both the photon source and the detectors have large inefficiencies, in general we still have inefficient 
(exponential) scaling for construction of large-scale graph states, even after the above improvement [23]. If one wants to generate an $n$-qubit graph-state entanglement, one needs to consume $n / 2$ imperfect entangled pairs represented by the state (1) and detect $n$ photon modes at the end. So, there is a factor of $\eta_{d}^{n} \eta_{s}^{n / 2}$ in the preparation efficiency, where $\eta_{d}$ is the efficiency for each individual detector. If we need $m(m \leq n / 2)$ PBS gates to arrive at such a graph state, there is also additional factor of $(1 / 2)^{m}$ in the preparation efficiency associated with the intrinsic gate success probability (to stay in the subspace $S$ ). In the case of a small source efficiency $\eta_{s}$ (such as for the SPDC experiments), the preparation efficiency goes down pretty quickly with the size of the state, which limits the current implementation to a small number of qubits [1, 2, 3, 4, 5]. In the following, we will show that for a subclass of graph-state entanglement, that is, for any $n$-qubit entanglements represented by tree graph states, we can prepare and detect them very efficiently with a polynomial scaling over the state size.

The achievement of this efficient scaling is based on a combination of the ideas of the divide-and-conquer (quantum repeater) protocol and the postselection measurements. We note that for applications of graph states in linear optics quantum information, each photon mode needs to be eventually measured in some polarization basis. This suggests that the whole protocol can be divided into two logical steps: the graph state preparation and the application measurements. For the second step, measurement of each photon mode has a finite failure probability, where instead of getting the photon's polarization, one does not register any photon. To boost the efficiency of the whole protocol, it is better to sort out and discard these failure events as soon as possible. In this spirit, we can try to apply the applications measurements on some individual qubits before we finish the first logic step of the graph-state preparation. We measure the qubits as soon as we do not need to apply the PBS gates on those qubits any more. When we register a failure event, we immediately discard the qubits that are influenced by the failure event, and restart the state preparation for that segment.

Figure 3 illustrates how such an idea works for preparation and detection of a tree-graph state. We start with two pairs $(1,2)$ and $(3,4)$, with the pair state described by Eq. (1). As we do not need to apply the PBS gates on the qubits 1 and 4 in the following steps, we immediately measure them in the polarization basis chosen according to the targeted application protocol. The measurement on the qubit 1 (or 4) succeeds with a probability $p_{0}=\eta_{s} \eta_{d}$, and upon a success, the vacuum component in the imperfect state (1) is eliminated. If we fail for the measurement on the qubit 1 , we only need to re-prepare the state for the pair $(1,2)$, with the pair $(3,4)$ intact [24]. With in average $1 / p_{0}$ trials, we succeed to get a good effective state (without the vacuum component) for the pair $(1,2)$. Parallel to this effort, we also get a good state for the pair $(3,4)$, similarly with $1 / p_{0}$ trials. Then,

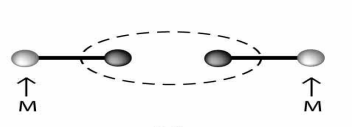

[a]

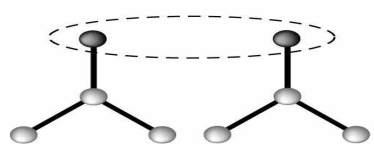

[c]

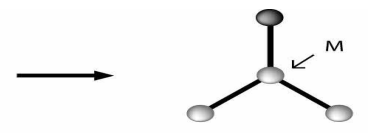

[b]

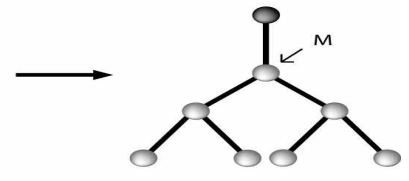

[d]
FIG. 3: Efficient construction of tree-graph states. White circles represent qubits that have have been measured in appropriate polarization bases, and black circles represent the connection qubits (unmeasured) which enable the next-step connection. [a] Before connection of the two center qubits, the two edge qubits have been measured. [b] After connection, we immediately measure one of the connection qubits, and leave the other one for the next step connection as shown in [c]. [c-d] Repeat the process of connection-and-measurement for construction of larger graphs.

we continue with the connection of the qubits 2 and 3 through a PBS gate, and after the connection, we immediately measure the qubit 2 as we only need keep the qubit 3 for the next step of connection. This process is continued until we get an effective tree-graph state with a desired number of qubits.

To figure out the overall efficiency for generation of this graph-state entanglement, we need to specify the recursion relations for each step of connection. For each connection, the number of qubits is doubled. For the $m$ th connection, the effective state before connection can be written as $\rho_{2 n}^{a}=\rho_{n} \otimes \rho_{n}$, where $\rho_{n}$ is the state of a segment which has $n=2^{m}$ qubits. The segment state can be expressed as $\rho_{n}=a_{m-1} \rho_{g}+\left(1-a_{m-1}\right) \rho_{\text {vac }}$, where $\rho_{g}$ denotes the effective $n$-qubit tree graph state and $\rho_{\text {vac }}$ represents the vacuum component where the connection qubit of the graph is in the vacuum state. For the 1st connection (the connection of the pair $(1,2)$ and $(3,4)$ ), as the vacuum component has been eliminated by the measurement on the qubits 1 and 4 , we have $a_{0}=1$. After the $m$ th connection, we immediately measure one of the two connection qubits (the other one is kept as the connection qubit for the next step). The success probability for this measurement is given by

$p_{m}=\eta_{d}\left[a_{m-1}^{2} / 2+a_{m-1}^{2}\left(2-\eta_{d}\right) / 4+a_{m-1}\left(1-a_{m-1}\right)\right]$,

where we have assumed the detector cannot distinguish the single-photon and two-photon counts, as it is the case in practice. Upon a success of this measurement, the effective state for the $2 n$ qubits becomes $\rho_{2 n}=$ $a_{m} \rho_{g}+\left(1-a_{m}\right) \rho_{\text {vac }}$, where $\rho_{g}$ and $\rho_{\text {vac }}$ have the same meaning as before except that they are for $2 n$ qubits now, and the coefficient $a_{m}$ is given by the recursion relation $a_{m}=2 a_{m-1} /\left(4-\eta_{d} a_{m-1}\right)$. Together with $a_{0}=1$, this 
recursion relation yields

$$
a_{m}=\left[2^{m}\left(1-\eta_{d} / 2\right)+\eta_{d} / 2\right]^{-1} .
$$

To prepare and confirm an $n=2^{m}$ qubit entanglement represented by the tree graph state, the overall efficiency of the scheme can be characterized by the total preparation time $T$. From the above recursion relations, one can find that

$$
\begin{aligned}
T & =t_{0}\left(\eta_{d} a_{m-1}\right)^{-1} \prod_{i=0}^{m-1}\left(1 / p_{i}\right) \\
& \approx t_{0}\left(\eta_{s} \eta_{d}\right)^{-1} n^{\left[\left(\log _{2} n-1\right) / 2+\log _{2}\left(1 / \eta_{d}-1 / 2\right)\right]},
\end{aligned}
$$

where the approximation is valid when $\eta_{d} / 2 \ll n$, and we have assumed that the two segments of graphs states before each connection can be prepared in parallel. The $t_{0}$ in $T$ denotes the time to generate the imperfect pair (1), which is basically the inverse of the pulse repetition rate in the SPDC experiment [1, 2, 3, 4]. On can see that $T$ scales nearly polynomially with the size $n$ of the final graph state, and such a scaling holds for any positive source efficiency $\eta_{s}$ and detector efficiency $\eta_{d}$.

Before ending the manuscript, let us briefly mention some practical implications of this method. If we take the source efficiency $\eta_{s} \sim 1 \%$ and the detector efficiency $\eta_{d} \sim 70 \%$, as it is typical for current experiments [1, 2, 3, 4], we get $T / t_{0} \sim 1.8 \times 10^{8}$ for preparation of a graph state of 128 qubits. If the pulse repetition rate is 80 $\mathrm{MHz}$ (the value from the SPDC experiment [1, 2, 3, 4]), the total preparation time $T$ will be about 2 seconds, which is still pretty reasonable. If we do not use this divide-and conquer technique, the total time will be given by $T / t_{0}=\eta_{s}^{-64} \eta_{d}^{-128} 2^{128 / 2-1} \sim 10^{167}$ for entanglement of 128 qubits, which is terribly long. We should also mention that we neglect in this paper other sources of noise except the photon source and detector inefficiencies. This is well justified as other noise is typically much smaller in magnitudes. For instance, the dark count probability within the pulse interval $(\sim 1 / 80 \mu s)$ is below $10^{-4}$, which indicates such a noise is negligible for entanglement of hundreds of qubits.

In summary, we have shown that a single polarization beam splitter acts as a powerful gate in the physical subspace postselected by the photon detection. Such a gate is very efficient for generating graph state of any shapes. In particular, we have shown for preparation and detection of tree graph states, the required resources scale only polynomially with size of the graph. This result opens up a prospect to generate large scale entanglement with the state-of the-art technology, while such entanglement is useful for implementation of various quantum information protocols.

This work was supported by the NSF awards (0431476), the ARDA under ARO contracts, and the A. P. Sloan Fellowship.
[1] P. G. Kwiat et al., Phys. Rev. Lett. 75, 4337 (1995).

[2] D. Bouwmeester et al., Nature 390, 575 (1997); D. Boschi et al., Phys. Rev. Lett. 80, 1121 (1998).

[3] J.-W. Pan, et al., Phys. Rev. Lett. 86, 4435-4438 (2001).

[4] P.Walther, et al., Nature 434, 169 (2005); N. Kiesel et al, Phys. Rev. Lett. 95, 210502 (2005).

[5] T.B. Pittman, B.C Jacobs, J.D. Franson, quant-ph/0408093

[6] E. Knill, R. Laflamme, and G. Milburn, Nature 409, 46 (2001).

[7] N. Yoran and B. Reznik, Phys. Rev. Lett. 91, 037903 (2003).

[8] M.A. Nielsen, Phys. Rev. Lett. 93, 040503 (2004).

[9] D.E. Browne, T. Rudolph, Phys. Rev. Lett. 95, 010501 (2005).

[10] M. Varnava, D. E. Browne, T. Rudolph, quant-ph/0507036

[11] C. M. Dawson, H. L. Haselgrove, M. A. Nielsen, Phys. Rev. Lett. 96, 020501 (2006).

[12] L. M. Duan, M. D. Lukin, J. I. Cirac, P,. Zoller, Nature 414, 413 (2001).

[13] H.-J. Briegel, W. Duer, J. I. Cirac, P. Zoller, Phys. Rev. Lett. 81, 5932 (1998).

[14] L.-M. Duan, Phys. Rev. Lett. 88, 170402 (2002).

[15] To achieve efficient scaling for quantum communication through the divide-and-conquer method, the photon source and detector efficiencies can be any positive number, which is equivalent to say that the threshold efficiency is $0 \%$ for this particular noise model.
[16] A. Kuzmich, et al., Nature 423, 731 (2003); T. Chanelire, et al., Nature 438, 833 (2005); M. D. Eisaman, et al., Nature 438, 837 (2005).

[17] H.J. Briegel and R. Raussendorf, Phys. Rev. Lett. 86, 910 (2001); R. Raussendorf and H. J. Briegel, Phys. Rev. Lett. 86, 5188 (2001).

[18] M. Hein et al., quant-ph/0602096

[19] Note that in the type-I or type-II fusion gate [9], one detects one or two output modes, which means that one has to discard one or more photons for each gate operation.

[20] Y. Shi, L.-M. Duan, G. Vidal, quant-ph/0511193

[21] L.-M. Duan, J. Kimble, Phys. Rev. Lett. 90, 253601 (2003); ibid 92, 127902 (2004).

[22] B. B. Blinov, D. L. Moehring, L.-M. Duan, C. Monroe, Nature 428, 153-157 (2004).

[23] If we assume perfect detectors and photon source, we can construct any graph state with an efficient scaling through the PBS gate with the method of Refs. [8, 9]. In this work, however, we would like to insist on a more realistic assumption with large inefficiencies for both of the photon source and the detectors.

[24] We assumed here that we can keep the pair $(3,4)$ for a little while to wait for the success of the measurement on the qubit 1. Such a memory (a controllable delay) is an essential requirement for scalability of all linear optics quantum information protocols, see [5, 16, 22] for some experiments in this direction. 